Multimodal analysis of resting state cortical activity: what does fMRI add to our knowledge of microstates in resting state EEG activity? Commentary to the papers by Britz et al. and Musso et al. in the current issue of NeuroImage

Lehmann, D

DOI: https://doi.org/10.1016/j.neuroimage.2010.05.033

Posted at the Zurich Open Repository and Archive, University of Zurich ZORA URL: https://doi.org/10.5167/uzh-42140

Journal Article

Accepted Version

Originally published at:

Lehmann, D (2010). Multimodal analysis of resting state cortical activity: what does fMRI add to our knowledge of microstates in resting state EEG activity? Commentary to the papers by Britz et al. and Musso et al. in the current issue of NeuroImage. NeuroImage, 52(4):1173-1174.

DOI: https://doi.org/10.1016/j.neuroimage.2010.05.033 
As published in:

NeuroImage 52[4]: 1173-1174 (2010).

PMID:20493265

Comments and Controversies

\section{Multimodal analysis of resting state cortical activity: What does fMRI add to our knowledge of microstates in resting state EEG activity?}

\section{Commentary to the papers by Britz et al. and Musso et al. in the current issue of NeuroImage}

\section{Dietrich Lehmann}

E-mail address: dlehmann@key.uzh.ch.

URL: http://www.uzh.ch/keyinst/.

The KEY Institute for Brain-Mind Research, University Hospital of Psychiatry, Zurich, Switzerland

The functional state of living systems changes in a wide range of time scales: There are developmental changes from birth to death, daily wake-sleep cycles, fluctuations of attention in the range of seconds, and split-second states compatible with the speed of thoughts. EEG wave-shape oriented analysis captures spontaneous changes down to the second range (Berger, 1929) where resting (no task, no stimulus) state EEG has well-known norms, and systematic deviations from those norms are observed in specific macrostates of perception, cognition, attention, development, vigilance, drug conditions, and pathology (e.g., John et al., 1988; Niedermeyer and Lopes da Silva, 2005). The claims about the function of resting states and their disturbances in the more recent fMRI literature parallel these known EEG findings. However, the type of description of the resting states obtained from EEG and fMRI are very different (see, e.g., Laufs et al., 2006). Space-oriented EEG analysis yields state descriptions with millisecond time resolution. This approach was utilized in the two independent papers by Britz et al., 2010; Musso et al., 2010 in the current issue of NeuroImage.In fMRI-EEG coregistrations and coanalyses, the two papers demonstrate that fMRI resting states reflect certain aspects of EEG microstates in the split-second range. The two papers showed that EEG functional microstates (Lehmann et al., 1987, 1998, 2009) correlate with the BOLD response in different brain areas, and that the spatial patterns of these responses resemble some of the well-known resting state networks. The two studies thus indicate that EEG microstates and fMRI resting states measure at least partially common processes. The exciting novelty of this finding is that it establishes a link between connectivity at different time scales: EEG microstates represent split-second, transiently synchronous neuronal activity, while the much slower fMRI resting state networks represent metabolic coactivation. In other words, the functional "glue" that assembles the metabolic resting state networks observed in fMRI may be highly synchronous neuronal activity, as has been postulated as a potential binding mechanism in animal studies (see also Jann et al., 2009).

The partial correlation of task-induced fMRI activation patterns and the resting state networks had led to the conclusion that functional networks relevant to information processing also are spontaneously activated at rest (e.g., Mantini et al., 2007). This is also the case for the EEG. It has been shown that maps evoked by specific stimuli are similar to those that are activated during specific types of spontaneous mentation; more concretely, core EEG networks that are activated after presentation of nouns with high vs. low imagery content were the same as those activated during spontaneous visual vs. abstract thoughts (Lehmann et al., 2010). 
EEG microstates (Lehmann, 1971; Lehmann et al., 1987, 1998, 2009) refer to the transiently stable topography (spatial configuration) of the scalp potential field measured with multichannel EEG, and result from the discontinuous, collective activity of large-scale neuronal networks in the brain. Changes of the topography of themomentary scalp potential maps are due to changes in the configuration of the active network. In spontaneous EEG, these topography changes appear approximately every 80-120 milliseconds. In otherwords, a given topography remains stable for about 100 milliseconds, indicating a highly coherent global brain state in this split-second range (Koenig et al., 2005). The duration of about 100 milliseconds matches the time of discontinuous motor or perceptual processes or the duration needed to establish long-distance synchrony (Harter, 1967; Efron, 1970; Di Lollo, 1980; Varela et al., 2001; Pockett et al., 2009). The observation of temporal segments of spatial stability corresponds well to the assumption that a stream of discrete units rather than a continuous flow of neuronal activity characterizes brain information processing (Baars, 2002; Lehmann and Skrandies, 1980; Sergent and Dehaene, 2004). These discrete units do not only appear during stimulus processing but are also observed during spontaneous conscious thinking (James, 1890; Newell, 1992). Indeed, the spatial configuration of the spontaneous microstates has been previously shown to covary with the type of conscious thoughts, suggesting that functional microstates are the basic building blocks of mentation, the "atoms of thought" (Lehmann et al., 1998). There is also a temporal structure of the microstates: Their duration as well as their transition probability (their syntax) is not random (Wackermann et al., 1993; Lehmann et al., 2005); they followcertain rules that yet remain to be understood. Incidentally, discontinuous behavior of brain activity during no-task conditions has also been observed with very different analysis approaches (Breakspear et al., 2004; Freeman et al., 2003; Kitzbichler et al. 2009).

It has to be kept inmind that the EEGcan only give a blurred image of the actual neuronal source distribution and is relatively insensitive to deep sources, while the fMRI can only give a blurred account of the temporal dynamics of brain activity and is exclusively sensitive only to slow fluctuations. Therefore, the complementary virtues and shortcomings of rfMRI and EEG technologies suggest that combining the two approaches would be most valuable to have information about both, the temporal structure and the spatial distribution of the resting state networks that are present in a given experimental or clinical condition. The contributions by Britz et al., 2010; Musso et al., 2010 represent important advances in this respect.

\section{References}

Baars, B.J., 2002. Behaviorism redux? Trends Cogn. Sci. 6 (6), 268-269.

Berger, H., 1929. Über das Elektroenkephalogramm des Menschen. Arch. Psychiatr. Nervenkr. 87, 527-570.

Breakspear,M.,Williams, L.M., Stam, C.J., 2004. Topographic analysis of phase dynamics in neural systems reveals formation and dissolution of "dynamic cell assemblies". J. Comput. Neurosci. 16, 49-68.

Britz, J., Van De Ville, D., Michel, C.M., 2010. BOLD correlates of EEG topography reveal rapid resting-state network dynamics. NeuroImage 52, 1162-1170.

Di Lollo, V., 1980. Temporal integration in visual memory. J. Exp. Psychol. Gen. 109 (1), 75-97.

Efron, E., 1970. The minimum duration of a perception. Neuropsychologia 8 (1), 57-63.

Freeman, W.J., Burke, B.C., Holmes, M.D., 2003. Aperiodic phase re-setting in scalp EEG of beta-gamma oscillations by state transitions at alpha-theta rates. Hum. Brain Mapp. 19 (4), 248-272.

Harter,M.R., 1967. Excitability cycles and cortical scanning: a review of two hypotheses of central intermittency in perception. Psychol. Bull. 68 (1), 47-58.

James, W., 1890. The Principles of Psychology, Vol. 1. Dover, New York. 
Jann, K., Dierks, T., Boesch, C., Kottlow, M., Strik,W., Koenig, T., 2009. BOLD correlates of EEG alpha phase-locking and the fMRI default mode network. NeuroImage 45 (3), 903916.

John, E.R., Prichep, L.S., Fridman, J., Easton, P., 1988. Neurometrics: computer-assisted differential diagnosis of brain dysfunctions. Science 239 (4836), 162-169.

Kitzbichler,M.G., Smith, M.L., Christensen, S.R., Bullmore, E., 2009. Broadband criticality of human brain network synchronization. PLoS Comput. Biol. 5 (3), e1000314

Koenig, T., Studer, D., Hubl, D.,Melie, L., Strik,W.K., 2005. Brain connectivity at different time-scales measured with EEG. Philos. Trans. R. Soc. Lond. B Biol. Sci. 360 (1457), $1015-1023$.

Laufs,H., Holt, J.L., Elfont, R., Krams,M., Paul, J.S., Krakow, K., Kleinschmidt, A., 2006. Where the BOLD signal goes when alpha EEG leaves. NeuroImage 31 (4), 14081418.

Lehmann, D., 1971. Multichannel topography of human alpha EEG fields. Electroenceph. Clin. Neurophysiol. 31 (5), 439-449.

Lehmann, D., Skrandies, W., 1980. Reference-free identification of components of checkerboard-evokedmultichannel potential fields. Electroenceph. Clin.Neurophysiol. 48 (6), 609-621.

Lehmann, D., Ozaki, H., Pal, I., 1987. EEG alpha map series: brain micro-states by spaceoriented adaptive segmentation. Electroenceph. Clin. Neurophysiol. 67 (3), 271-288.

Lehmann, D., Strik, W.K., Henggeler, B., Koenig, T., Koukkou, M., 1998. Brain electric microstates and momentary conscious mind states as building blocks of spontaneous thinking: I. Visual imagery and abstract thoughts. Int. J. Psychophysiol. 29 (1), 1-11.

Lehmann, D., Faber, P.L., Galderisi, S., Herrmann,W.M., Kinoshita, T., Koukkou, M., Mucci, A., Pascual-Marqui, R.D., Saito, N.,Wackermann, J.,Winterer, G., Koenig, T., 2005. EEG microstate duration and syntax in acute, medication-naïve, first-episode schizophrenia: a multi-center study. Psychiatr. Res. NeuroImaging 138 (2), 141-156.

Lehmann, D., Pascual-Marqui, R.D., Michel, C., 2009. EEG microstates. Scholarpedia 4 (3), 7632 URL: http://www.scholarpedia.org/article/EEG_microstates.

Lehmann, D., Pascual-Marqui, R.D., Strik, W.K., Koenig, T., 2010. Core networks for visualconcrete and abstract thought content: a brain electric microstate analysis. Neuroimage 49 (1), 1073-1079.

Mantini, D., Perrucci, M.G., Del Gratta, C., Romani, G.L., Corbetta, M., 2007. Electrophysiological signatures of resting state networks in the human brain. Proc. Natl Acad. Sci. USA 104 (32), 13170-13175.

Musso, F., Brinkmeyer, J., Mobascher, A., Warbrick, T., Winterer, G., 2010. Spontaneous brain activity and EEG microstates. A novel EEG/fMRI analysis approach to explore resting-state networks. NeuroImage 52, 1149-1161.

Newell, A., 1992. Précis of unified theories of cognition. Behav. Brain Sci. 15, 425-492.

Niedermeyer, E., Lopes da Silva, F., 2005. Electroencephalography: Basic Principles, Clinical Applications, and Related Fields5th ed. Wiley, New York.

Pockett, S., Bold, G.E., Freeman, W.J., 2009. EEG synchrony during a perceptual-cognitive task: widespread phase synchrony at all frequencies. Clin. Neurophysiol. 120 (4), 695-708.

Sergent, C., Dehaene, S., 2004. Neural processes underlying conscious perception: experimental findings and a global neuronal workspace framework. J. Physiol. Paris 98 (46), 374-384.

Varela, F., Lachaux, J.P., Rodriguez, E., Martinerie, J., 2001. The brainweb: phase synchronization and large-scale integration. Nat. Rev. Neurosci. 2 (4), 229-239.

Wackermann, J., Lehmann, D.,Michel, C.M., Strik,W.K., 1993. Adaptive segmentation of spontaneous EEGmap series into spatially definedmicrostates. Int. J. Psychophysiol. 14 (3), 269-283. 